\title{
On Level-Set Type Methods for Recovering Piecewise Constant Solutions of Ill-Posed Problems
}

\author{
Adriano DeCezaro ${ }^{1}$, Antonio Leitão ${ }^{2}$, and Xue-Cheng Tai ${ }^{3,4}$ \\ 1 Institute of Mathematics Statistics and Physics, Federal University of Rio Grande, \\ Av. Italia km 8, 96201-900 Rio Grande, Brazil \\ decezaro@impa.br \\ 2 Department of Mathematics, Federal University of St. Catarina, P.O. Box 476, \\ 88040-900 Florianópolis, Brazil \\ acgleitao@gmail.com \\ 3 Division of Mathematical Science, School of Physical and Mathematical Sciences, \\ Nanyang Technological University, Singapore \\ xctai@ntu.edu.sg \\ 4 Department of Mathematics, University of Bergen, Johannes Brunsgate 12, \\ N-5008 Bergen, Norway
}

\begin{abstract}
We propose a regularization method for solving ill-posed problems, under the assumption that the solutions are piecewise constant functions with unknown level sets and unknown level values. A level set framework is established for the inverse problem and a Tikhonov regularization approach is proposed. Existence of generalized minimizers for the Tikhonov functional is proven. Moreover, we establish convergence and stability results, characterizing our Tikhonov approach as a regularization method. Based on the necessary conditions of optimality for the Tikhonov functional, a level-set type method is derived and implemented numerically for solving an inverse source problem. This allow us to test the quality of the proposed algorithm.
\end{abstract}

\section{Introduction}

Several inverse problems of interest consist of identifying an unknown physical quantity $u \in X$, that can be represented by a piecewise constant function, over a bounded given domain $\Omega$, from the set of data $y \in Y$, where $X, Y$ are Hilbert spaces. This process being described by the model

$$
F(u)=y,
$$

where $F: D(F) \subset X \rightarrow Y$ and the set of data is obtained by indirect measurements of the parameter. Because of this, in practical applications the exact data $y \in Y$ is, in general, not known. Given is only approximate measured data $y^{\delta} \in Y$, corrupted by noise of level $\delta>0$ and satisfying

$$
\left\|y^{\delta}-y\right\|_{Y} \leq \delta
$$

X.-C. Tai et al. (Eds.): SSVM 2009, LNCS 5567, pp. 50-62, 2009.

(C) Springer-Verlag Berlin Heidelberg 2009 
In the case where the unknown function $u$ is a piecewise constant function distinguishing between two given values (i.e., up to an affine transformation, $u$ is a characteristic function), level set approaches were considered in $[1,2,3,4,5,6$. In this case, since the level values of $u$ are known, one needs only to identify the level sets of $u$, i.e. the inverse problem reduces to a shape identification problem.

In the case where the unknown function $u$ is a piecewise constant function distinguishing between several given values, multiple level set approaches were considered in [6,78. If the level values of $u$ are also unknown the inverse problem becomes harder, since one has to identify both the level sets as well as the level values of the unknown parameter $u$. In this case, the dimension of the parameter space increases by the number of unknown level values.

Our starting point in this article is the assumption that the parameter $u$ in (1) is a piecewise constant function assuming two unknown values, i.e. $u(x) \in$ $\left\{c^{1}, c^{2}\right\}$ a.e. in $\Omega \subset \mathbb{R}^{d}, d=2,3$. In this case one can assume the existence of an open mensurable set $D \subset \subset \Omega$ s.t. $u(x)=c^{1}, x \in D$ and $u(x)=c^{2} x \in \Omega / D$.

We propose a level set approach to represent the unknown parameter $u$. First we introduce the $H^{1}$-function $\phi$, which act as a regularization on the parameter space. Then, using the Heaviside projector $H: H^{1}(\Omega) \rightarrow L^{\infty}(\Omega)$, a solution of (1) can be represented in the form

$$
u=c^{1} H(\phi)+c^{2}(1-H(\phi))=: P\left(\phi, c^{1}, c^{2}\right) .
$$

With this notation we have $D=\{x \in \Omega ; \phi(x)>0\}$ and $\Omega / D=\{x \in \Omega ; \phi(x)<$ $0\}$. The level values $c^{1}, c^{2} \in \mathbb{R}$ are unknown and have to be determined as well.

As already observed in [3], the Heaviside operator $H$ maps $H^{1}(\Omega)$ into the set $\mathcal{V}:=\left\{\chi_{D} ; D \subset \Omega\right.$ measurable, $\left.\mathcal{H}^{n-1}(\partial D)<\infty\right\}$, where $\mathcal{H}^{n-1}(S)$ denotes the (n-1)-dimensional Hausdorff-measure of the set $S$. Therefore, the operator $P$ in (3) maps $H^{1}(\Omega) \times R^{2}$ into the admissible parameter set $\mathcal{U}:=\left\{u=q\left(v, c^{1}, c^{2}\right)\right.$; $v \in \mathcal{V}$ and $\left.c^{1}, c^{2} \in \mathbb{R}\right\}$, where $q: \mathcal{V} \times \mathbb{R}^{2} \ni\left(v, c^{1}, c^{2}\right) \mapsto c^{1} v+c^{2}(1-v) \in L^{\infty}(\Omega)$.

Using the level set framework introduced above, the inverse problem in (1), with data given as in (2), can be written in the form of the operator equation

$$
F\left(P\left(\phi, c^{1}, c^{2}\right)\right)=y^{\delta} .
$$

Once an approximate solution $\left(\phi, c^{1}, c^{2}\right)$ of (4) is obtained, a corresponding solution of (11) can be computed using equation (3). In this article, approximate solutions to (4) are obtained by minimizing the Tikhonov functional

$$
\begin{array}{r}
\mathcal{G}_{\alpha}\left(\phi, c^{1}, c^{2}\right):=\left\|F\left(P\left(\phi, c^{1}, c^{2}\right)\right)-y^{\delta}\right\|_{Y}^{2}+\alpha\left\{\beta_{1}|H(\phi)|_{\mathrm{BV}}+\beta_{2}\left\|\phi-\phi_{0}\right\|_{H^{1}}^{2}\right. \\
\left.+\beta_{3} \sum_{j=1}^{2}\left|c^{j}-c_{0}^{j}\right|^{2}\right\},
\end{array}
$$

based on $T V-H^{1}$ penalization. Here $\phi_{0}$ and $c_{0}^{j}$ are known reference parameters. This Tikhonov functional extends the ones proposed in [5, 6, 9] (based on $T V$ penalization) and [3, 8] (based on $T V-H^{1}$ penalization). To motivate the regularization terms in (5), notice that they effect: i) the boundedness of the level lines of $\phi$ as well as it's $H^{1}$-norm; ii) the boundedness of $c_{j}$. These two facts allow us to guarantee existence of (generalized) minimizers of $\mathcal{G}_{\alpha}$ in $L^{\infty} \cap \mathrm{BV}$. 
This article is outlined as follows: In Section 2 we introduce the concept of generalized minimizers for the functional $\mathcal{G}_{\alpha}$ in (5). In Section 3 we derive a convergence analysis for this Tikhonov approach. In Section 4 we introduce stabilized functionals and prove that the corresponding minimizers approximate a minimizer of $\mathcal{G}_{\alpha}$. Section 5 is devoted to numerical experiments. A level set type method is implemented for solving a two-dimensional inverse potential problem.

\section{The Concept of Generalized Minimizers}

We shall consider the model problem described as in the introduction under the following general assumptions:

(A1) $\Omega \subseteq \mathbb{R}^{n}$ is bounded, connected, with piecewise $C^{1}$ boundary $\partial \Omega$.

(A2) The operator $F: D(F) \subset L^{1}(\Omega) \rightarrow Y$ is continuous and Fréchetdifferentiable on $D(F)$ with respect to the $L^{1}(\Omega)$-topology.

(A3) $\varepsilon, \alpha$ and $\beta_{j}, j=1,2,3$ denote positive parameters.

(A4) Equation (11) has a solution, i.e. there exists $u \in \mathcal{U}$ satisfying $F(u)=y$ and a function $\phi \in H^{1}(\Omega)$ satisfying $|\nabla \phi| \neq 0$, in a neighborhood of $\{\phi=0\}$ such that $H(\phi)=z$, for some $z \in \mathcal{V}$. Moreover, there exist constants values $c^{1}, c^{2} \in \mathbb{R}$ such that $q\left(z, c^{1}, c^{2}\right)=u$.

For each $\varepsilon>0$, we define the operator

$$
P_{\varepsilon}\left(\phi, c^{1}, c^{2}\right):=c^{1} H_{\varepsilon}(\phi)+c^{2}\left(1-H_{\varepsilon}(\phi)\right),
$$

where $H_{\varepsilon}$ is the continuous approximation to $H$ given by:

$$
H_{\varepsilon}(t):=\left\{\begin{array}{rl}
1+t / \varepsilon & \text { for } t \in[-\varepsilon, 0] \\
H(t) & \text { for } t \in \mathbb{R} /[-\varepsilon, 0]
\end{array} .\right.
$$

In order to guarantee existence of a minimizer of $\mathcal{G}_{\alpha}$ in (5), we adapt to the level-set framework described above, the concept of generalized minimizers formulated in [3].

Definition 1. Let the operators $H, P, H_{\varepsilon}$ and $P_{\varepsilon}$ be defined as above.

a) $A$ vector $\left(z, \phi, c^{1}, c^{2}\right) \in L^{\infty}(\Omega) \times H^{1}(\Omega) \times \mathbb{R}^{2}$ is called admissible when there exists a sequence $\left\{\phi_{k}\right\}$ of $H^{1}(\Omega)$-functions satisfying $\lim _{k}\left\|\phi_{k}-\phi\right\|_{L^{2}}=$ 0 , and also there exists a sequence $\left\{\varepsilon_{k}\right\} \in \mathbb{R}^{+}$converging to zero such that $\lim _{k}\left\|H_{\varepsilon_{k}}\left(\phi_{k}\right)-z\right\|_{L^{1}}=0$.

b) $A$ minimizer of $\mathcal{G}_{\alpha}$ is considered to be any admissible vector $\left(z, \phi, c^{1}, c^{2}\right)$ minimizing

$$
\mathcal{G}_{\alpha}\left(z, \phi, c^{1}, c^{2}\right):=\left\|F\left(q\left(z, c^{1}, c^{2}\right)\right)-y^{\delta}\right\|_{Y}^{2}+\alpha R\left(z, \phi, c^{1}, c^{2}\right)
$$

over the set of admissible vectors, where

$$
\begin{gathered}
R\left(z, \phi, c^{1}, c^{2}\right)=\rho(z, \phi)+\beta_{3} \sum_{j=1}^{2}\left|c^{j}-c_{0}^{j}\right|^{2}, \\
\rho(z, \phi):=\inf \left\{\liminf _{k \rightarrow \infty}\left[\beta_{1}\left|H_{\varepsilon_{k}}\left(\phi_{k}\right)\right|_{\mathrm{BV}}+\beta_{2}\left\|\phi_{k}-\phi_{0}\right\|_{H^{1}}^{2}\right]\right\} .
\end{gathered}
$$

The infimum in (9) is taken over all sequences $\left\{\varepsilon_{k}\right\}$ and $\left\{\phi_{k}\right\}$ characterizing $\left(z, \phi, c^{1}, c^{2}\right)$ as an admissible vector. 
c) A generalized minimizer of $\mathcal{G}_{\alpha}\left(\phi, c^{1}, c^{2}\right)$ is an admissible vector $\left(z, \phi, c^{1}, c^{2}\right)$ minimizing the functional $\mathcal{G}_{\alpha}$ in (7) on the set of admissible vectors.

\subsection{Relevant Properties of Admissible Vectors}

First we verify some basic properties of the operators $P_{\varepsilon}, H_{\varepsilon}$ and $q$ that will be necessary in the subsequent analysis.

Lemma 1. Let $\Omega$ be given as above and $j=1,2$.

(i) Let $\left\{z_{k}\right\}_{k \in \mathbb{N}}$ be a bounded sequence in $L^{\infty}(\Omega)$ converging to some element $z$ in $L^{1}(\Omega)$ and $\left\{c_{k}^{j}\right\}_{k \in \mathbb{N}}$ be a sequence of real numbers converging to $c^{j}$. Then $q\left(z_{k}, c_{k}^{1}, c_{k}^{2}\right)$ converges to $q\left(z, c^{1}, c^{2}\right)$ in $L^{1}(\Omega)$.

(ii) Let $(z, \phi) \in L^{1}(\Omega) \times H^{1}(\Omega)$, be such that $H_{\varepsilon}(\phi) \rightarrow z$ in $L^{1}(\Omega)$ as $\varepsilon \rightarrow 0$ and let $c^{1}, c^{2} \in \mathbb{R}$. Then $P_{\varepsilon}\left(\phi, c^{1}, c^{2}\right) \rightarrow q\left(z, c^{1}, c^{2}\right)$ in $L^{1}(\Omega)$ as $\varepsilon \rightarrow 0$.

(iii) Given $\varepsilon>0$, let $\left\{\phi_{k}\right\}_{k \in \mathbb{N}}$ be a sequence in $H^{1}(\Omega)$ converging to $\phi \in H^{1}(\Omega)$ in the $L^{2}$-norm. Then $H_{\varepsilon}\left(\phi_{k}\right) \rightarrow H_{\varepsilon}(\phi)$ in $L^{1}(\Omega)$, as $k \rightarrow \infty$. Moreover, if $\left\{c_{k}^{j}\right\}_{k \in \mathbb{N}}$ are sequences of real numbers converging to some $c^{j}$, then $q\left(H_{\varepsilon}\left(\phi_{k}\right), c_{k}^{1}, c_{k}^{2}\right) \rightarrow q\left(H_{\varepsilon}(\phi), c^{1}, c^{2}\right)$ in $L^{1}(\Omega)$, as $k \rightarrow \infty$.

Proof. Since $\Omega$ is assumed to be bounded, we have $L^{\infty}(\Omega) \subset L^{1}(\Omega)$. To prove (i), notice that

$$
\begin{aligned}
& \left\|q\left(z_{k}, c_{k}^{1}, c_{k}^{2}\right)-q\left(z, c^{1}, c^{2}\right)\right\|_{L^{1}}=\int_{\Omega}\left|c_{k}^{1} z_{k}+c_{k}^{2}\left(1-z_{k}\right)-c^{1} z-c^{2}(1-z)\right| d x \\
& \leq \int_{\Omega}\left|z_{k}\right|\left(\left|c_{k}^{1}-c^{1}\right|+\left|c_{k}^{2}-c^{2}\right|\right) d x+\int_{\Omega}\left(\left(\left|c^{1}\right|+\left|c^{2}\right|\right)\left|z_{k}-z\right|+\left|c_{k}^{2}-c^{2}\right|\right) d x \\
& \leq|\Omega|\left\|z_{k}\right\|_{L^{\infty}}\left(\left|c_{k}^{1}-c^{1}\right|+\left|c_{k}^{2}-c^{2}\right|\right)+\left(\left|c^{1}\right|+\left|c^{2}\right|\right)\left\|z_{k}-z\right\|_{L^{1}}+|\Omega|\left|c_{k}^{2}-c^{2}\right|,
\end{aligned}
$$

which converges to zero as $k \rightarrow \infty$. Assertion (ii) follows with similar arguments. The first part of assertion (iii) is a direct consequence of the inequality $\| H_{\varepsilon}\left(\phi_{k}\right)-$ $H_{\varepsilon}(\phi)\left\|_{L^{1}(\Omega)} \leq \varepsilon^{-1} \sqrt{\operatorname{meas}(\Omega)}\right\| \phi_{k}-\phi \|_{L^{2}(\Omega)}$. The second part of assertion (iii) follows by a combination of the inequality above and assertion (i).

Lemma 2. Let $\left(z_{k}, \phi_{k}, c_{k}^{1}, c_{k}^{2}\right)$ be a sequence of admissible vectors converging in $L^{1}(\Omega) \times L^{2}(\Omega) \times \mathbb{R}^{2}$ to some $\left(z, \phi, c^{1}, c^{2}\right)$. Then $\left(z, \phi, c^{1}, c^{2}\right)$ is also an admissible vector.

Sketch of the proof. In order to prove that $\left(z, \phi, c^{1}, c^{2}\right)$ is also an admissible vector, one uses an argument of extraction of diagonal subsequences, analogously as in [8, Lemma 2].

\subsection{Relevant Properties of the Penalization Functional}

In the next lemmas we verify two properties of the functional $R$ which are fundamental for the convergence analysis in Section 3

Lemma 3. The functional $R$ in (8) is coercive on the set of admissible vectors. 
Sketch of the proof. Let $\left(z, \phi, c^{1}, c^{2}\right)$ be an admissible vector. From [8, Lemma 4] follows

$$
\rho(z, \phi) \geq\left(\beta_{1}|z|_{\mathrm{BV}}+\beta_{2}\left\|\phi-\phi_{0}\right\|_{H^{1}}^{2}\right) .
$$

Now, from (10) and the definition of $R$ in (8) follows

$$
\begin{aligned}
\beta_{1}|z|_{\mathrm{BV}}+\beta_{2}\left\|\phi-\phi_{0}\right\|_{H^{1}}^{2}+\beta_{3} \sum_{j=1}^{2}\left|c^{j}-c_{0}^{j}\right|^{2} \leq \\
\rho(z, \phi)+\beta_{3} \sum_{j=1}^{2}\left|c^{j}-c_{0}^{j}\right|^{2}=R\left(z, \phi, c^{1}, c^{2}\right),
\end{aligned}
$$

concluding the proof.

Lemma 4. The functional $R$ in (8) is weak lower semi-continuous on the set of admissible vectors, i.e. given a sequence $\left\{\left(z_{k}, \phi_{k}, c_{k}^{1}, c_{k}^{2}\right)\right\}$ of admissible vectors such that $z_{k} \rightarrow z$ in $L^{1}(\Omega), \phi_{k} \rightarrow \phi$ in $H^{1}(\Omega), c_{k}^{j} \rightarrow c^{j}$ in $\mathbb{R}$, for some admissible vector $\left(z, \phi, c^{1}, c^{2}\right)$, then it follows

$$
R\left(z, \phi, c^{1}, c^{2}\right) \leq \liminf _{k \in \mathbb{N}} R\left(z_{k}, \phi_{k}, c_{k}^{1}, c_{k}^{2}\right) .
$$

Proof. The functional $\rho(z, \phi)$ is weak lower semi-continuous cf. [8, Lemma 5]. Moreover, the Euclidean norm in $\mathbb{R}^{2}$ is also lower semi-continuous. The lemma follows from the fact that the functional $R$ in (8) is a linear combination of lower semi-continuous functionals.

\section{Convergence Analysis}

First we prove that for any positive parameters $\alpha, \beta$ the functional $\mathcal{G}_{\alpha}$ in (5) is well posed.

Theorem 1 (Well-Posedness). The functional $\mathcal{G}_{\alpha}$ in (5) attains minimizers on the set of admissible vectors.

Proof. Notice that the set of admissible vectors is not empty, since $(0,0,0,0)$ is admissible. Let $\left\{\left(z_{k}, \phi_{k}, c_{k}^{1}, c_{k}^{2}\right)\right\}$ be a minimizing sequence for $\mathcal{G}_{\alpha}$, i.e. a sequence of admissible vectors satisfying $\mathcal{G}_{\alpha}\left(z_{k}, \phi_{k}, c_{k}^{1}, c_{k}^{2}\right) \rightarrow \inf \mathcal{G}_{\alpha} \leq \mathcal{G}_{\alpha}(0,0,0,0)<\infty$. Then, $\left\{\mathcal{G}_{\alpha}\left(z_{k}, \phi_{k}, c_{k}^{1}, c_{k}^{2}\right)\right\}$ is a bounded sequence of real numbers. Therefore, $\left\{\left(z_{k}, \phi_{k}, c_{k}^{1}, c^{2}, k\right)\right\}$ is uniformly bounded in $\mathrm{BV} \times H^{1}(\Omega) \times \mathbb{R}^{2}$. Thus, the Sobolev compact embedding theorem 10 and the Bolzano-Weierstrass theorem guarantees the existence of a subsequence (denoted again by $\left\{\left(z_{k}, \phi_{k}, c_{k}^{1}, c_{k}^{2}\right)\right\}$ ) and the existence of $\left(z, \phi, c^{1}, c^{2}\right) \in L^{1}(\Omega) \times H^{1}(\Omega) \times \mathbb{R}^{2}$ such that $\phi_{k} \rightarrow \phi$ in $L^{2}(\Omega)$, $\phi_{k} \rightarrow \phi$ in $H^{1}(\Omega), z_{k} \rightarrow z$ in $L^{1}(\Omega)$ and $c_{k}^{j} \rightarrow c^{j}$ in $\mathbb{R}$.

From Lemma 2 we conclude that $\left(z, \phi, c^{1}, c^{2}\right)$ is an admissible vector. Moreover, from the weak lower semi-continuity of $R$ together with the continuity of $F$ and $q$ we obtain

$$
\begin{gathered}
\lim _{k \rightarrow \infty} \mathcal{G}_{\alpha}\left(z_{k}, \phi_{k}, c_{k}^{1}, c_{k}^{2}\right)=\lim _{k \rightarrow \infty}\left\{\left\|F\left(q\left(z_{k}, c_{k}^{1}, c_{k}^{2}\right)\right)-y^{\delta}\right\|_{Y}^{2}+\alpha R\left(z_{k}, \phi_{k}, c_{k}^{1}, c_{k}^{2}\right)\right\} \\
\geq\left\|F\left(q\left(z, c^{1}, c^{2}\right)\right)-y^{\delta}\right\|_{Y}^{2}+\alpha R\left(z, \phi, c^{1}, c^{2}\right)=\mathcal{G}_{\alpha}\left(z, \phi, c^{1}, c^{2}\right),
\end{gathered}
$$

proving that $\left(z, \phi, c^{1}, c^{2}\right)$ minimizes $\mathcal{G}_{\alpha}$. 
In the next theorems we present the main convergence and stability results. The proofs use classical techniques from the analysis of Tikhonov type regularization methods (see, e.g., [11,12]).

Theorem 2 (Convergence for exact data). Assume that we have exact data, i.e. $y^{\delta}=y$ and $\beta_{j}>0, j=1,2,3$. For every $\alpha>0$ let $\left(z_{\alpha}, \phi_{\alpha}, c_{\alpha}^{1}, c_{\alpha}^{2}\right)$ denote a minimizer of $\mathcal{G}_{\alpha}$ on the set of admissible vectors. Then, for every sequence of positive numbers $\left\{\alpha_{k}\right\}_{k \in \mathbb{N}}$ converging to zero there exists a subsequence, denoted again by $\left\{\alpha_{k}\right\}_{l \in \mathbb{N}}$, such that $\left(z_{\alpha_{k}}, \phi_{\alpha_{k}}, c_{\alpha_{k}}^{1}, c_{\alpha_{k}}^{2}\right)$ is strongly convergent in $L^{1}(\Omega) \times$ $L^{2}(\Omega) \times \mathbb{R}^{2}$. Moreover, the limit is a solution of (1).

Proof. Let $\left(z^{\dagger}, \phi^{\dagger}, c^{1, \dagger}, c^{2, \dagger}\right)$ be a solution of (1) - its existence is guaranteed by assumption (A4). Let $\left\{\alpha_{k}\right\}_{k \in \mathbb{N}}$ be a sequence of positive numbers converging to zero. For each $k \in \mathbb{N}$, let $\left(z_{k}, \phi_{k}, c_{k}^{1}, c_{k}^{2}\right):=\left(z_{\alpha_{k}}, \phi_{\alpha_{k}}, c_{\alpha_{k}}^{1}, c_{\alpha_{k}}^{2}\right)$ be a minimizer of $G_{\alpha_{k}}$. Then, for each $k \in \mathbb{N}$ we have

$$
\begin{aligned}
G_{\alpha_{k}}\left(z_{k}, \phi_{k}, c_{k}^{1}, c_{k}^{2}\right) \leq\left\|F\left(q\left(z^{\dagger}, c^{1, \dagger}, c^{2, \dagger}\right)\right)-y\right\|_{Y}^{2} & +\alpha_{k} R\left(z^{\dagger}, \phi^{\dagger}, c^{1, \dagger}, c^{2, \dagger}\right) \\
& =\alpha_{k} R\left(z^{\dagger}, \phi^{\dagger}, c^{1, \dagger}, c^{2, \dagger}\right) .
\end{aligned}
$$

Since $\alpha_{k} R\left(z_{k}, \phi_{k}, c_{k}^{1}, c_{k}^{2}\right) \leq G_{\alpha_{k}}\left(z_{k}, \phi_{k}, c_{k}^{1}, c_{k}^{2}\right)$, it follows from (12) that

$$
R\left(z_{k}, \phi_{k}, c_{k}^{1}, c_{k}^{2}\right) \leq R\left(z^{\dagger}, \phi^{\dagger}, c^{1, \dagger}, c^{2, \dagger}\right)<\infty .
$$

Moreover, from the assumption on the sequence $\left\{\alpha_{k}\right\}$, it follows that

$$
\lim _{k \rightarrow \infty} \alpha_{k} R\left(z^{\dagger}, \phi^{\dagger}, c^{1, \dagger}, c^{1, \dagger}\right)=0
$$

From (13) and Lemma 3 we conclude that the sequences $\left\{\phi_{k}\right\},\left\{z_{k}\right\}$ and $\left\{c_{k}^{j}\right\}^{j=1,2}$ are bounded in $H^{1}(\Omega)$, BV and $\mathbb{R}^{2}$ respectively. Using an argument of extraction of diagonal subsequences (see proof of Lemma 2) we can guarantee the existence of an admissible vector $\left(\tilde{z}, \tilde{\phi}, \tilde{c}^{1}, \tilde{c}^{2}\right)$ such that

$$
\left(z_{k}, \phi_{k}, c_{k}^{1}, c_{k}^{2}\right) \rightarrow\left(\tilde{z}, \tilde{\phi}, \tilde{c}^{1}, \tilde{c}^{2}\right) \text { in } L^{1}(\Omega) \times L^{2}(\Omega) \times \mathbb{R}^{2} .
$$

From Lemma 1 (i) follows that $q\left(\tilde{z}, \tilde{c}^{1}, \tilde{c}^{2}\right)=\lim _{k \rightarrow \infty} q\left(z_{k}, c_{k}^{1}, c_{k}^{2}\right)$ on $L^{1}(\Omega)$. Using the continuity of the operator $F$ together with (12) and (14) we conclude that

$$
y=\lim _{k \rightarrow \infty} F\left(q\left(z_{k}, c_{k}^{1}, c_{k}^{2}\right)\right)=F\left(q\left(\tilde{z}, \tilde{c}^{1}, \tilde{c}^{2}\right)\right) .
$$

On the other hand, from the lower semi-continuity of $R$ and (13) it follows that

$$
\begin{aligned}
R\left(\tilde{z}, \tilde{\phi}, \tilde{c}^{1}, \tilde{c}^{2}\right) \leq \liminf _{k \rightarrow \infty} R\left(z_{k}, \phi_{k}, c_{k}^{1}, c_{k}^{2}\right) & \\
& \left.\leq \limsup _{k \rightarrow \infty} R\left(z_{k}, \phi_{k}, c_{k}^{1}, c_{k}^{2}\right)\right) \leq R\left(z^{\dagger}, \phi^{\dagger}, \tilde{c}^{1}, \tilde{c}^{2}\right),
\end{aligned}
$$

concluding the proof. 
Theorem 3 (Convergence for noisy data). Let $\alpha=\alpha(\delta)$ be a function satisfying $\lim _{\delta \rightarrow 0} \alpha(\delta)=0$ and $\lim _{\delta \rightarrow 0} \delta^{2} \alpha(\delta)^{-1}=0$. Moreover, let $\left\{\delta_{k}\right\}_{k \in \mathbb{N}}$ be a sequence of positive numbers converging to zero and $y^{\delta_{k}} \in Y$ be corresponding noisy data satisfying (21). Then, there exist a subsequence, denoted again by $\left\{\delta_{k}\right\}$, and a sequence $\left\{\alpha_{k}:=\alpha\left(\delta_{k}\right)\right\}$ such that $\left(z_{\alpha_{k}}, \phi_{\alpha_{k}}, c_{\alpha_{k}}^{1}, c_{\alpha_{k}}^{2}\right)$ converges in $L^{1}(\Omega) \times L^{2}(\Omega) \times \mathbb{R}^{2}$ to solution of (1).

Proof. Let $\left(z^{\dagger}, \phi^{\dagger}, c^{1, \dagger}, c^{1, \dagger}\right)$ be a solution of (11) 1 For each $k \in \mathbb{N}$, denote by $\left(z_{k}, \phi_{k}, c_{k}^{1}, c_{k}^{2}\right):=\left(z_{\alpha\left(\delta_{k}\right)}, \phi_{\alpha\left(\delta_{k}\right)}, c_{\alpha\left(\delta_{k}\right)}^{1}, c_{\alpha\left(\delta_{k}\right)}^{2}\right)$ a minimizer of $G_{\alpha\left(\delta_{k}\right)}$. Then, for each $k \in \mathbb{N}$ we have

$$
\begin{aligned}
G_{\alpha_{k}}\left(z_{k}, \phi_{k}, c_{k}^{1}, c_{k}^{2}\right) & \leq\left\|F\left(q\left(z^{\dagger}, c^{1, \dagger}, c^{1, \dagger}\right)\right)-y^{\delta_{k}}\right\|_{Y}^{2}+\alpha\left(\delta_{k}\right) R\left(z^{\dagger}, \phi^{\dagger}, c^{1, \dagger}, c^{2, \dagger}\right) \\
& \leq \delta_{k}^{2}+\alpha\left(\delta_{k}\right) R\left(z^{\dagger}, \phi^{\dagger}, c^{1, \dagger}, c^{2, \dagger}\right) .
\end{aligned}
$$

Taking the limit $k \rightarrow \infty$ in (15), it follows from the theorem assumptions that $\lim _{k \rightarrow \infty}\left\|F\left(q\left(z_{k}, c_{k}^{1}, c_{k}^{2}\right)\right)-y^{\delta_{k}}\right\|^{2} \leq \lim _{k \rightarrow \infty} G_{\alpha_{k}}\left(z_{k}, \phi_{k}, c_{k}^{1}, c_{k}^{2}\right)=0$. Therefore, $\lim _{k \rightarrow \infty} F\left(q\left(z_{k}, c_{k}^{1}, c_{k}^{2}\right)\right)=y$. Moreover, from (15) and the definition of $G_{\alpha_{k}}$, it follows that $R\left(z_{k}, \phi_{k}, c_{k}^{1}, c_{k}^{2}\right) \leq \delta_{k}^{2} \alpha\left(\delta_{k}\right)^{-1}+R\left(z^{\dagger}, \phi^{\dagger}, c^{1, \dagger}, c^{2, \dagger}\right)$. Thus, from the assumptions on the function $\alpha\left(\delta_{k}\right)$, we conclude that $\limsup R\left(z_{k}, \phi_{k}, c_{k}^{1}, c_{k}^{2}\right) \leq$ $R\left(z^{\dagger}, \phi^{\dagger}, c^{1, \dagger}, c^{2, \dagger}\right)$. The proof follows arguing as in the proof of Lemma 2

\section{Numerical Solution}

In the sequel we introduce a functional which can be handled numerically, and whose minimizers are 'close' to the minimizers of $\mathcal{G}_{\alpha}$. Let $\mathcal{G}_{\varepsilon, \alpha}$ be the stabilized functional defined by

$$
\begin{aligned}
\mathcal{G}_{\varepsilon, \alpha}\left(\phi, c^{1}, c^{2}\right):=\| F\left(P_{\varepsilon}\left(\phi, c^{1}, c^{2}\right)\right) & -y^{\delta} \|_{Y}^{2}+\alpha\left\{\beta_{1}\left|H_{\varepsilon}(\phi)\right|_{\mathrm{BV}}+\right. \\
& \left.+\beta_{2}\left\|\phi-\phi_{0}\right\|_{H^{1}}^{2}+\beta_{3} \sum_{j=1}^{2}\left|c^{j}-c_{0}^{j}\right|^{2}\right\},
\end{aligned}
$$

where $P_{\varepsilon}\left(\phi, c^{1}, c^{2}\right):=q\left(H_{\varepsilon}(\phi), c^{1}, c^{2}\right)$ is the functional defined in (6). The functional $\mathcal{G}_{\varepsilon, \alpha}$ is well-posed as the following lemma shows:

Lemma 5. Given positive constants $\alpha, \varepsilon, \beta_{j}, j=1,2,3$ as above, a function $\phi_{0} \in H^{1}(\Omega)$ and $c_{0}^{j} \in \mathbb{R}, j=1,2$, the functional $\mathcal{G}_{\varepsilon, \alpha}$ in (16) attains a minimizer on $H^{1}(\Omega) \times \mathbb{R}^{2}$.

Proof. Since $\inf \left\{\mathcal{G}_{\varepsilon, \alpha}\left(\phi, c^{1}, c^{2}\right):\left(\phi, c^{1}, c^{2}\right) \in H^{1}(\Omega) \times \mathbb{R}^{2}\right\} \leq \mathcal{G}_{\varepsilon, \alpha}(0,0,0)<\infty$, there exists a minimizing sequence $\left\{\left(\phi_{k}, c_{k}^{1}, c_{k}^{2}\right)\right\}$ in $H^{1}(\Omega) \times \mathbb{R}^{2}$ satisfying

$$
\lim _{k \rightarrow \infty} \mathcal{G}_{\varepsilon, \alpha}\left(\phi_{k}, c_{k}^{1}, c_{k}^{2}\right)=\inf \left\{\mathcal{G}_{\varepsilon, \alpha}\left(\phi, c^{1}, c^{2}\right):\left(\phi, c^{1}, c^{2}\right) \in H^{1}(\Omega) \times \mathbb{R}^{2}\right\} .
$$

$\overline{1}$ The existence of solutions is guaranteed by (A4). 
Then, for fixed $\alpha>0$, the sequences $\left\{\phi_{k}\right\}$ and $\left\{c_{k}^{j}\right\}^{j=1,2}$ are bounded in $H^{1}(\Omega)$ and $\mathbb{R}^{2}$ respectively. Therefore, $\phi_{k} \rightarrow \phi$ in $H^{1}(\Omega)$ and $c_{k}^{j} \rightarrow c^{j}$ in $\mathbb{R}, j=1,2$. Moreover, by the weak lower semi-continuity of the $H^{1}$-norm and the continuity of the Euclidean norm in $\mathbb{R}$, it follows that $\left\|\phi-\phi_{0}\right\|_{H^{1}}^{2} \leq \liminf _{k \rightarrow \infty}\left\|\phi_{k}-\phi_{0}\right\|_{H^{1}}^{2}$, and $\left|c^{j}-c_{0}^{j}\right| \leq \liminf _{k \rightarrow \infty}\left|c_{k}^{j}-c_{0}^{j}\right|$.

From the Sobolev compact embedding theorem [13] we have $\phi_{k} \rightarrow \phi$ in $L^{2}(\Omega)$. Therefore, Lemma 1 implies

$$
\begin{aligned}
\left\|H_{\varepsilon}\left(\phi_{k}^{j}\right)-H_{\varepsilon}\left(\phi^{j}\right)\right\|_{L^{1}} & \leq \varepsilon^{-1} \sqrt{\operatorname{meas}}(\Omega)\left\|\phi_{k}-\phi\right\|_{L^{2}} \rightarrow 0, \\
\left\|P_{\varepsilon}\left(\phi_{k}, c_{k}^{1}, c_{k}^{2}\right)-P_{\varepsilon}\left(\phi, c^{1}, c^{2}\right)\right\|_{L^{1}} & =\left\|q\left(H_{\varepsilon}\left(\phi_{k}\right), c_{k}^{1}, c_{k}^{2}\right)-q\left(H_{\varepsilon}(\phi), c^{1}, c^{2}\right)\right\|_{L^{1}} \rightarrow 0 .
\end{aligned}
$$

Thus, it follows from [10, Theorem 1, pg 172] that $\left|H_{\varepsilon}(\phi)\right|_{\mathrm{BV}} \leq \liminf _{k \rightarrow \infty}\left|H_{\varepsilon}\left(\phi_{k}\right)\right|_{\mathrm{BV}}$. Now, from the continuity of $F$ and $q$, together with the estimates above we obtain

$$
\begin{aligned}
\mathcal{G}_{\varepsilon, \alpha}\left(\phi, c^{1}, c^{2}\right) \leq & \lim _{k \rightarrow \infty}\left\|F\left(P_{\varepsilon}\left(\phi_{k}, c_{k}^{1}, c_{k}^{2}\right)\right)-y^{\delta}\right\|_{Y}^{2}+\alpha\left\{\beta_{1} \liminf _{k \rightarrow \infty}\left|H_{\varepsilon}\left(\phi_{k}\right)\right|_{\mathrm{BV}}+\right. \\
& \left.+\beta_{2} \liminf _{k \rightarrow \infty}\left\|\phi_{k}-\phi_{0}\right\|_{H^{1}}^{2}+\beta_{3} \liminf _{k \rightarrow \infty} \sum_{j=1}^{2}\left|c_{k}^{j}-c_{0}^{j}\right|^{2}\right\} \\
\leq & \liminf _{k \rightarrow \infty} \mathcal{G}_{\varepsilon, \alpha}\left(\phi_{k}, c_{k}^{1}, c_{k}^{2}\right)=\inf \mathcal{G}_{\varepsilon, \alpha},
\end{aligned}
$$

concluding the proof.

In the sequel we prove that, when $\varepsilon \rightarrow 0$, the minimizers of $\mathcal{G}_{\varepsilon, \alpha}$ approximate a minimizer of the functional $\mathcal{G}_{\alpha}$.

Theorem 4. Let $\alpha$ and $\beta_{j}$ be given as above. For each $\varepsilon>0$, denote by $\left(\phi_{\varepsilon, \alpha}\right.$, $\left.c_{\varepsilon, \alpha}^{1}, c_{\varepsilon, \alpha}^{2}\right)$ a minimizer of $\mathcal{G}_{\varepsilon, \alpha}$. There exists a sequence of positive numbers $\varepsilon_{k} \rightarrow$ 0 such that $\left(H_{\varepsilon_{k}}\left(\phi_{\varepsilon_{k}, \alpha}\right), \phi_{\varepsilon_{k}, \alpha}, c_{\varepsilon_{k}, \alpha}^{1}, c_{\varepsilon_{k}, \alpha}^{2}\right)$ converges strongly in $L^{1}(\Omega) \times L^{2}(\Omega) \times$ $\mathbb{R}^{2}$ and the limit minimizes $\mathcal{G}_{\alpha}$ on the set of admissible vectors.

Proof. The functional $\mathcal{G}_{\alpha}$ attains a generalized minimizer $\left(z_{\alpha}, \phi_{\alpha}, c_{\alpha}^{1}, c_{\alpha}^{2}\right)$ on the set of admissible vectors (cf. Theorem 1). From Definition 1, there exists a sequence $\left\{\varepsilon_{k}\right\}$ of positive numbers converging to zero and corresponding sequences $\left\{\phi_{k}\right\}$ in $H^{1}(\Omega)$ satisfying $\phi_{k} \rightarrow \phi_{\alpha}$ in $L^{2}(\Omega), H_{\varepsilon_{k}}\left(\phi_{k}\right) \rightarrow z_{\alpha}$ in $L^{1}(\Omega)$. Moreover, we can further assume [8, Lemma 3] that

$R\left(z_{\alpha}, \phi_{\alpha}, c_{\alpha}^{1}, c_{\alpha}^{1}\right)=\lim _{k \rightarrow \infty}\left\{\beta_{1}\left|H_{\varepsilon_{k}}\left(\phi_{k}\right)\right|_{\mathrm{BV}}+\beta_{2}\left\|\phi_{k}-\phi_{0}\right\|_{H^{1}}^{2}+\beta_{3} \sum_{j=1}^{2}\left|c_{k}^{j}-c_{0}^{j}\right|^{2}\right\}$.

Let $\left(\phi_{\varepsilon_{k}}, c_{\varepsilon_{k}}^{1}, c_{\varepsilon_{k}}^{2}\right)$ be a minimizer of $\mathcal{G}_{\varepsilon_{k}, \alpha}$. The sequences $\left\{\phi_{\varepsilon_{k}}\right\},\left\{H_{\varepsilon_{k}}\left(\phi_{\varepsilon_{k}}\right)\right\}$ and $\left\{c_{k}^{j}\right\}^{j=1,2}$ are uniformly bounded in $H^{1}(\Omega), \operatorname{BV}(\Omega)$ and $\mathbb{R}^{2}$ respectively. By the compact Sobolev embedding theorem [13, the compact embedding of BV into $L^{1}$ [10] and the Bolzano-Weierstrass theorem, there exist convergent subsequences whose limits are denoted by $\tilde{\phi}, \tilde{z}$ and $\tilde{c}^{j}$. Summarizing, we have $\phi_{\varepsilon_{k}} \rightarrow \tilde{\phi}$ in $L^{2}(\Omega), H_{\varepsilon_{k}}\left(\phi_{\varepsilon_{k}}\right) \rightarrow \tilde{z}$ in $L^{1}(\Omega)$, and $c_{k}^{j} \rightarrow \tilde{c}^{j}$ in $\mathbb{R}, j=1,2$. Thus, $\left(\tilde{z}, \tilde{\phi}, \tilde{c}^{1}, \tilde{c}^{2}\right) \in$ $L^{1}(\Omega) \times H^{1}(\Omega) \times \mathbb{R}^{2}$ is an admissible vector (cf. Lemma 2 ). 
From the definition of $R$, Lemma 1 and the continuity of $F$, it follows that

$$
\begin{gathered}
\left\|F\left(q\left(\tilde{z}, \tilde{c}^{1}, \tilde{c}^{2}\right)\right)-y^{\delta}\right\|_{Y}^{2}=\lim _{k \rightarrow \infty}\left\|F\left(P_{\varepsilon_{k}}\left(\phi_{\varepsilon_{k}}, c_{\varepsilon_{k}}^{1}, c_{\varepsilon_{k}}^{2}\right)\right)-y^{\delta}\right\|_{Y}^{2}, \\
R\left(\tilde{z}, \tilde{\phi}, \tilde{c}^{1}, \tilde{c}^{2}\right) \leq \liminf _{k \rightarrow \infty}\left\{\beta_{1}\left|H_{\varepsilon_{k}}\left(\phi_{\varepsilon_{k}}\right)\right|_{\mathrm{BV}}+\beta_{2}\left\|\phi_{\varepsilon_{k}}-\phi_{0}\right\|_{H^{1}}^{2}+\beta_{3} \sum_{j=1}^{2}\left|c_{\varepsilon_{k}}^{j}-c_{0}^{j}\right|^{2}\right\} .
\end{gathered}
$$

Therefore,

$$
\begin{aligned}
& \mathcal{G}_{\alpha}\left(\tilde{z}, \tilde{\phi}, \tilde{c}^{1}, \tilde{c}^{2}\right)=\left\|F\left(q\left(\tilde{z}, \tilde{c}^{1}, \tilde{c}^{2}\right)\right)-y^{\delta}\right\|_{Y}^{2}+\alpha R\left(\tilde{z}, \tilde{\phi}, \tilde{c}^{1}, \tilde{c}^{2}\right) \\
& \leq \liminf _{k \rightarrow \infty} \mathcal{G}_{\varepsilon_{k}, \alpha}\left(\phi_{\varepsilon_{k}}, c_{\varepsilon_{k}}^{1}, c_{\varepsilon_{k}}^{2}\right) \leq \liminf _{k \rightarrow \infty} \mathcal{G}_{\varepsilon_{k}, \alpha}\left(\phi_{k}, c_{k}^{1}, c_{k}^{2}\right) \\
& \leq \limsup _{k \rightarrow \infty}\left\|F\left(P_{\varepsilon_{k}}\left(\phi_{k}, c_{k}^{1}, c_{k}^{2}\right)\right)-y^{\delta}\right\|_{Y}^{2} \\
& \quad+\alpha \limsup _{k \rightarrow \infty}\left\{\beta_{1}\left|H_{\varepsilon_{k}}\left(\phi_{k}\right)\right|_{\mathrm{BV}}+\beta_{2}\left\|\phi_{k}-\phi_{0}\right\|_{H^{1}}^{2}+\beta_{3} \sum_{j=1}^{2}\left|c_{k}^{j}-c_{0}^{j}\right|^{2}\right\} \\
& =\left\|F\left(q\left(z_{\alpha}, c_{\alpha}^{1}, c_{\alpha}^{2}\right)\right)-y^{\delta}\right\|_{Y}^{2}+\alpha R\left(z_{\alpha}, \phi_{\alpha}, c_{\alpha}^{1}, c_{\alpha}^{2}\right)=\mathcal{G}_{\alpha}\left(z_{\alpha}, \phi_{\alpha}^{1}, c_{\alpha}^{1}, c_{\alpha}^{2}\right),
\end{aligned}
$$

characterizing $\left(\tilde{z}, \tilde{\phi}, c_{\alpha}^{1}, c_{\alpha}^{2}\right)$ as a minimizer of $\mathcal{G}_{\alpha}$.

\subsection{Optimality Conditions for the Stabilized Functional}

For numerical purposes it is convenient to derive first order optimality conditions for minimizers of the stabilized functionals $\mathcal{G}_{\varepsilon, \alpha}$. Therefore, we consider $\mathcal{G}_{\varepsilon, \alpha}$ in (16) with $Y=L^{2}(\Omega)$ and we look for the Gâteaux directional derivatives with respect to $\phi$ and the unknown constants $c^{j}$ for $j=1,2$.

Since $H_{\varepsilon}^{\prime}(\phi)$ is self-adjoint, we can write the optimality conditions for the functional $\mathcal{G}_{\varepsilon, \alpha}$ in the form of the system

$$
\begin{gathered}
\alpha(\Delta-I)\left(\phi-\phi_{0}\right)=L_{\varepsilon, \alpha, \beta}\left(\phi, c^{1}, c^{2}\right), \text { in } \Omega ; \quad\left(\phi-\phi_{0}\right) \cdot \nu=0, \text { at } \partial \Omega \\
\alpha\left(c^{j}-c_{0}^{j}\right)=L_{\varepsilon, \alpha, \beta}^{j}\left(\phi, c^{1}, c^{2}\right), j=1,2 .
\end{gathered}
$$

Here $\nu(x)$ is the external unit normal vector at $x \in \partial \Omega, \bar{\beta}:=\left(2 \beta_{3}\right)^{-1}$, and

$$
\begin{array}{r}
L_{\varepsilon, \alpha, \beta}\left(\phi, c^{1}, c^{2}\right)=\left(c^{1}-c^{2}\right) \beta_{2}^{-1} H_{\varepsilon}^{\prime}(\phi)^{*} F^{\prime}\left(P_{\varepsilon}\left(\phi, c^{1}, c^{2}\right)\right)^{*}\left(F\left(P_{\varepsilon}\left(\phi, c^{1}, c^{2}\right)\right)-y^{\delta}\right) \\
-\beta_{1}\left(2 \beta_{2}\right)^{-1} H_{\varepsilon}^{\prime}(\phi) \nabla \cdot\left[\nabla H_{\varepsilon}(\phi) /\left|\nabla H_{\varepsilon}(\phi)\right|\right],(18 \mathrm{a}) \\
L_{\varepsilon, \alpha, \beta}^{1}\left(\phi, c^{1}, c^{2}\right)=\bar{\beta}\left(F^{\prime}\left(P_{\varepsilon}\left(\phi, c^{1}, c^{2}\right)\right) H_{\varepsilon}(\phi)\right)^{*}\left(F\left(P_{\varepsilon}\left(\phi, c^{1}, c^{2}\right)\right)-y^{\delta}\right), \quad(18 \mathrm{~b}) \\
L_{\varepsilon, \alpha, \beta}^{2}\left(\phi, c^{1}, c^{2}\right)=\bar{\beta}\left(F^{\prime}\left(P_{\varepsilon}\left(\phi, c^{1}, c^{2}\right)\right)\left(1-H_{\varepsilon}(\phi)\right)\right)^{*}\left(F\left(P_{\varepsilon}\left(\phi, c^{1}, c^{2}\right)\right)-y^{\delta}\right) .(18 \mathrm{c})
\end{array}
$$

\section{Numerical Results}

In this section a level-set type method based on the system of optimality conditions (17) is used for solving an inverse potential problem of recovering a piecewise constant function $u: \Omega \rightarrow\left\{c_{1}, c_{2}\right\}$, from measurements of the Cauchy data of its corresponding potential on the boundary of the domain $\Omega=(0,1) \times(0,1)$. Notice that no knowledge of the image of $u$ (values $c_{1}, c_{2} \in \mathbb{R}$ ) is assumed. 


\subsection{The Inverse Potential Problem}

To describe the direct problem, we define the operator $F: L^{2}(\Omega) \rightarrow L^{2}(\partial \Omega)$ by $F: u(x) \mapsto F(u):=\left.w_{\nu}\right|_{\partial \Omega}$, where $u$ is a piecewise constant function in $L^{2}(\Omega)$ with $u(x) \in\left\{c_{1}, c_{2}\right\}$ a.e. in $\Omega$, and $w \in H^{1}(\Omega)$ solves the elliptic boundary value problem

$$
\Delta w=u, \text { in } \Omega ; \quad w=0 \text {, at } \partial \Omega .
$$

Since $u \in L^{2}(\Omega)$, the Dirichlet boundary value problem in (19) has a unique solution, namely the potential $w \in H^{2}(\Omega) \cap H_{0}^{1}(\Omega)$.

The inverse problem we are concerned with, consists in determining the piecewise constant source function $u$ from measurements of the Neumann trace of $w$ at $\partial \Omega$, i.e. from $\left.w_{\nu}\right|_{\partial \Omega}$. Using the above notation, the inverse potential problem can be written in the abbreviated form $F(u)=y^{\delta}$, where the data $y^{\delta}$ has the same meaning as in (2).

Other inverse problems for the operator $F$ were considered in [3, 8]. In 3] a level set method was used for recovering the indicator function $u=\chi_{D}$ of a star-shaped domain $D \subset \mathbb{R}^{2}$. In [8] a multiple level set method was used for recovering a simple function $u: \Omega \rightarrow\left\{c_{1}, \ldots, c_{4}\right\}$. In both cases, knowledge of the (finite) image of $u$ was assumed.

\subsection{A Level-Set Algorithm for the Inverse Potential Problem}

In the sequel we describe the level set regularization algorithm. This method compares to the level set method as proposed in [8]. The complexity of our algorithm is as follows: at each iteration of the level set method, four elliptic boundary value problems (BVP) are solved (two of Dirichlet type and two of Neumann type).

In Table 1 an explicit fixed point procedure for solving the the optimality condition (18) is outlined. In the first step the residual $r_{k} \in L^{2}(\partial \Omega)$ of the iterate $\left(\phi_{k}, c_{k}^{1}, c_{k}^{2}\right)$ is evaluated. This corresponds to solving one elliptic BVP of Dirichlet type. In the second step the solution $h_{k} \in H^{1}(\Omega)$ of the adjoint problem for the residual is evaluated. This corresponds to solving one elliptic BVP of Dirichlet type. In the fourth step, the velocity function $v_{k} \in H^{1}(\Omega)$ for the level-set function is evaluated. This corresponds to solving an elliptic BVPs of Neumann type.

In the subsequent numerical experiments this algorithm was implemented using a finite element method for the solution of partial differential equations.

\subsection{Numerical Experiment}

In our experiment we consider the inverse problem of reconstructing the right hand side $u$ in (19) from the knowledge of a single pair of Cauchy data $\left(0, y^{\delta}\right)$ at $\partial \Omega$. We further assume that the level value $c_{2}=0$ is given, and that we have to identify only the support of $u$ and the level value $c_{1} \in \mathbb{R}^{+}$.

The data $y^{\delta}=y=F(u)$ for solving the inverse problem is known exactly, i.e. $\delta=0$, and is obtained by solving numerically the elliptic boundary value problem 
Table 1. Level set algorithm for the inverse potential problem

1. Evaluate the residual $r_{k}:=F\left(P_{\varepsilon}\left(\phi_{k}, c_{k}^{1}, c_{k}^{2}\right)\right)-y^{\delta}=\left.\left(w_{k}\right)_{\nu}\right|_{\partial \Omega}-y^{\delta}$, where $w_{k}$ solves

$$
\Delta w_{k}=P_{\varepsilon}\left(\phi_{k}, c_{k}^{1}, c_{k}^{2}\right), \text { in } \Omega ; \quad w_{k}=0, \text { at } \partial \Omega .
$$

2. Evaluate $h_{k}:=F^{\prime}\left(P_{\varepsilon}\left(\phi_{k}, c_{k}^{1}, c_{k}^{2}\right)\right)^{*}\left(r_{k}\right) \in L^{2}(\Omega)$, solving

$$
\Delta h_{k}=0, \text { in } \Omega ; \quad h_{k}=r_{k}, \text { at } \partial \Omega \text {. }
$$

3. Calculate $L_{\varepsilon, \alpha, \beta}\left(\phi_{k}, c_{k}^{1}, c_{k}^{2}\right)$ and $L_{\varepsilon, \alpha, \beta}^{j}\left(\phi_{k}, c_{k}^{1}, c_{k}^{2}\right), j=1,2$ as in (18).

4. Evaluate the velocity $v_{k} \in H^{1}(\Omega)$, solving

$$
(\Delta-I) v_{k}=L_{\varepsilon, \alpha, \beta}\left(\phi_{k}, c_{k}^{1}, c_{k}^{2}\right), \text { in } \Omega ; \quad\left(v_{k}\right)_{\nu}=0, \text { at } \partial \Omega .
$$

5. Update the level set function $\phi_{k}$ and the level values $c_{k}^{j}, j=1,2$ :

$$
\phi_{k+1}=\phi_{k}+\frac{1}{\alpha} v_{k}, \quad c_{k+1}^{j}=c_{k}^{j}+\frac{1}{\alpha} L_{\varepsilon, \alpha, \beta}^{j}\left(\phi_{k}, c_{k}^{1}, c_{k}^{2}\right) .
$$

in (19) (the word 'exactly' here means: up to the precision of the numerical method used for solving the direct problem).

For the direct problem we use the values: $c_{1}=1, c_{2}=0$ to compute the exact solution. In the computation of the inverse problem, the exact solution is known a priori to assume the values $\left\{c^{1}, 0\right\}$ (with unknown $c^{1}$ ). Moreover, when the data are given exactly, the iterative level-set method is implemented without the additional regularization term $\left|H_{\varepsilon}(\phi)\right|_{\mathrm{BV}}$, i.e. $\beta_{1}=0$.

The solution $u$ of the inverse problem as well as the initial guess $P_{\varepsilon}\left(\phi_{0}, c_{0}^{1}\right)$ for the level-set method are shown in Figure 1] Notice that the support of $u$ corresponds to a non-connected proper subset of $\Omega$, The initial guess $c_{0}^{1}=1.5$ is used for the unknown level value.

In Figure 2 the evolution of the level set method for the first 1500 iterative steps is presented. As one can see in this figure, the shapes of both inclusions are reasonably reconstructed, and the level value $c^{1}$ is accurately reconstructed as well. The iteration is stopped when the residual drops below the predefined precision $\left\|F\left(P_{\varepsilon}\left(\phi_{k}, c_{k}^{1}\right)\right)-y\right\|_{L^{2}}<10^{-2}$.
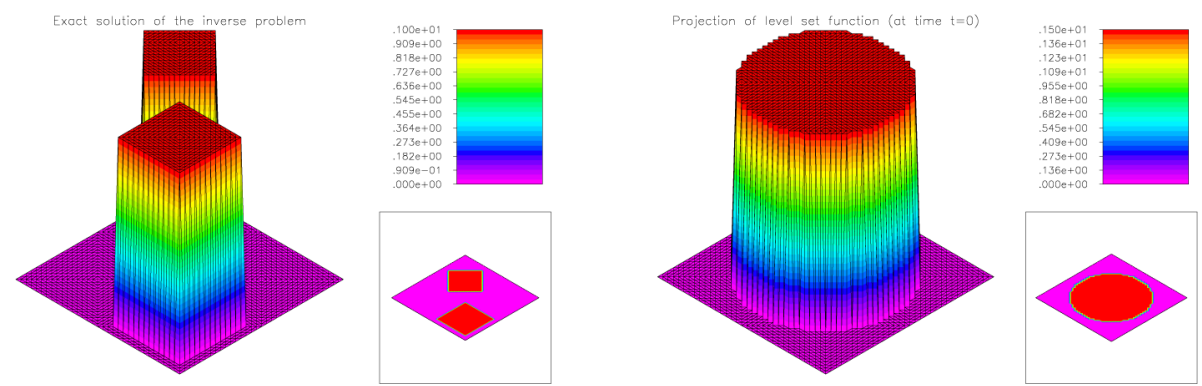

Fig. 1. Numerical experiment: The picture on the left hand side shows the coefficient to be reconstructed. On the right hand side, the initial condition for the level-set method. 

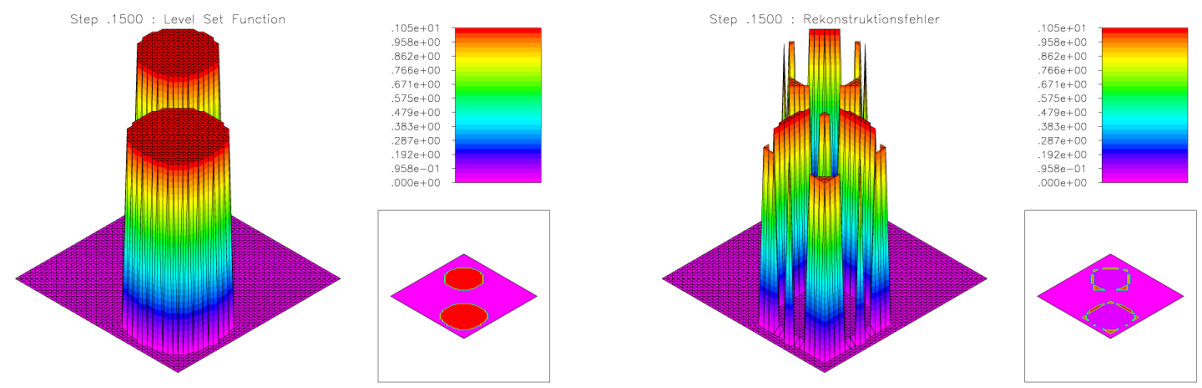

Fig. 2. Numerical experiment: On the left hand side a plot of $P\left(\phi_{k}, c_{k}^{1}\right)$ for $k=1500$. The picture on the right hand side shows the corresponding iteration error.

We performed other numerical simulations with different choice of initial guess $\left(\phi_{0}, c_{0}^{1}\right)$, and observed that the number of iterative steps required in order to obtain a reasonable approximation (up to the predefined precision of $10^{-2}$ in the $L^{2}$-norm) strongly depends on the choice of the initial guess $c_{0}^{1}$. On the other hand, the final result is not sensitive with respect to the choice of the initial guess $\phi_{0}$.

\section{Acknowledgments}

A.DC acknowledges the support from CNPq, grant 474593/2007-0. The work of A.L. is supported by the Brazilian National Research Council CNPq, grants 306020/2006-8, 474593/2007-0, and by the Alexander von Humbolt Foundation AvH. This article was written during a visit of the author to NTU (Singapore). X.-C.T. acknowledges the support from NTU SUG 20/07 and MOE Tier II project T207B2202 (ARC 29/07).

\section{References}

1. Santosa, F.: A level-set approach for inverse problems involving obstacles. ESAIM Contrôle Optim. Calc. Var. 1, 17-33 (1995/1996)

2. Leitão, A., Scherzer, O.: On the relation between constraint regularization, level sets, and shape optimization. Inverse Problems 19, L1-L11 (2003)

3. Frühauf, F., Scherzer, O., Leitão, A.: Analysis of regularization methods for the solution of ill-posed problems involving discontinuous operators. SIAM J. Numer. Anal. 43, 767-786 (2005)

4. Chung, E., Chan, T., Tai, X.C.: Electrical impedance tomography using level set representation and total variational regularization. J. Comput. Phys. 205(1), 357$372(2005)$

5. Chan, T., Tai, X.C.: Identification of discontinuous coefficients in elliptic problems using total variation regularization. SIAM J. Sci. Comput. 25(3), 881-904 (2003)

6. Chan, T., Tai, X.C.: Level set and total variation regularization for elliptic inverse problems with discontinuous coefficients. J. Comput. Phys. 193(1), 40-66 (2004) 
7. Chung, J., Vese, L.: Image segmantation using a multilayer level-sets apprach. UCLA C.A.M. Report 193(03-53), 1-28 (2003)

8. DeCezaro, A., Leitão, A., Tai, X.C.: On multiple level-set regularization methods for inverse problems. Inverse Problems 25 (to appear, 2009)

9. Tai, X.C., Chan, T.: A survey on multiple level set methods with applications for identifying piecewise constant functions. Int. J. Num. Anal. Model 1(1), 25-47 (2004)

10. Evans, L., Gariepy, R.: Measure theory and fine properties of functions. Studies in Advanced Mathematics. CRC Press, Boca Raton (1992)

11. Engl, H., Kunisch, K., Neubauer, A.: Convergence rates for Tikhonov regularisation of nonlinear ill-posed problems. Inverse Problems 5(4), 523-540 (1989)

12. Engl, H.W., Hanke, M., Neubauer, A.: Regularization of inverse problems. Mathematics and its Applications, vol. 375. Kluwer Academic Publishers Group, Dordrecht (1996)

13. Adams, R.: Sobolev Spaces. Academic Press, New York (1975) 